\title{
Behavioral activation for dementia caregivers: scheduling pleasant events and enhancing communications
}

\author{
Alma $\mathrm{Au}^{1,2}$ \\ Dolores Gallagher- \\ Thompson ${ }^{3}$ \\ Meng-Kong Wong ${ }^{4}$ \\ Jess Leung ${ }^{4}$ \\ Wai-Chi Chan ${ }^{5}$ \\ Chun Chung Chan ${ }^{6}$ \\ Hui-Jing Lu' \\ Man Kin Lai' \\ Kevin Chan' \\ 'Department of Applied Social \\ Sciences, ${ }^{2}$ Institute of Active Aging, \\ The Hong Kong Polytechnic University, \\ Hong Kong, People's Republic of China; \\ ${ }^{3}$ Department of Psychiatry and \\ Behavioral Sciences and Geriatric \\ Education Centre, School of Medicine, \\ Stanford University, Stanford, CA, USA; \\ ${ }^{4}$ Department of Psychiatry, United \\ Christian Hospital, ${ }^{5}$ Department of \\ Psychiatry, University of Hong \\ Kong, ${ }^{6}$ Department of Medicine and \\ Geriatrics, United Christian Hospital, \\ Hong Kong, People's Republic of China
}

Correspondence: Alma Au

Department of Applied Social Sciences, The Hong Kong Polytechnic University, II Yuk Choi Street, Hung Hom, Kowloon, Hong Kong, People's Republic of China

Tel +852 27667944

Emailssalma@polyu.edu.hk
This article was published in the following Dove Press journal:

Clinical Interventions in Aging

26 March 2015

Number of times this article has been viewed

Background: Dementia caregiving is often associated with increase in depressive symptoms and strained relationships. This study tested whether telephone-delivered psychoeducation combined with an enhanced behavioral activation (BA) module had a better effect on the well-being of Alzheimer's caregivers than psychoeducation alone. The focus is on enhancing the competent use of coping skills via BA. The program is delivered by telephone to increase accessibility and sustainability for caregivers. Senior citizens are trained as paraprofessionals to deliver the BA module to increase the potential for sustainability of the program.

Methods and subjects: The study compared two telephone interventions using a 4-month longitudinal randomized controlled trial. For the first 4 weeks, all participants received the same psychoeducation program via telephone. Then for the following 4 months, eight biweekly telephone follow-up calls were carried out. For these eight follow-up calls, participants were randomized into either one of the two following groups with different conditions. For the psychoeducation with BA (PsyED-BA) group, participants received eight biweekly sessions of BA practice focused on pleasant event scheduling and improving communications. For the psychoeducation only (PsyED only) group, participants received eight biweekly sessions of general discussion of psychoeducation and related information. A total of 62 family caregivers of persons living with dementia were recruited and 59 (29 in the PsyED-BA group and 30 in the PsyED only group) completed the whole study.

Results: As compared to the group with psychoeducation and discussion, the group with enhanced BA had decreased levels of depressive symptoms. The study had a low attrition rate.

Conclusion: Results suggested that competence-based training could be effectively administered through the telephone with the help of senior citizens trained and engaged as paraprofessionals. Results contribute to the present literature by offering some framework for developing effective, accessible, sustainable, and less costly interventions.

Keywords: telephone-assisted, paraprofessional, accessibility, sustainability

\section{Introduction}

Alzheimer's disease is regarded as the most prevalent of various types of dementia. According to the recent Alzheimer's Disease International report, the number of people living with dementia worldwide was estimated at 44 million in 2013, and is expected to reach 76 million by 2030 and 135 million by 2050 . In contrast to the previous finding that high-income countries have experienced the strongest upward trends, it will now be low- and middle-income countries which will feel the heaviest burden. ${ }^{1}$ By 2050 , $71 \%$ of dementia sufferers will live in low- and middle-income countries. Globally, many of these dementia patients are cared for by family members, who are referred to as informal caregivers. Converging evidence suggests that while informal caregiving 
can be rewarding, the burden of caring for a family member with dementia is associated with deteriorated physical health and reduced psychological well-being. ${ }^{2-6}$ However, caregiving can also be rewarding and strengthen family relationships. It is important to develop interventions that can significantly enhance the well-being of caregivers and reinforce the positive and uplifting experiences of caregiving. ${ }^{7-9}$

There is considerable worldwide concern to develop accessible, sustainable, and cost-effective interventions for caregivers to enable them to function and remain longer in the caregiving role in a more meaningful and productive and less stressful manner. ${ }^{10-13}$ A recent review by GallagherThompson et $\mathrm{al}^{2}$ has identified a variety of evidence-based nonpharmacological interventions on a global basis. These programs include the following: individual and family counseling, psychoeducation programs, specialized skill training, psychotherapy as well as interventions using technology such as telephone/smartphones and internet/online support. On the other hand, the pressing needs of family caregivers for help for themselves and their relatives often outstrip the available resources, even in developed countries. Moreover, the review also highlighted that it is not always simple or feasible to apply evidence-based or best practice programs developed in Western countries to other parts of the world due to cultural, economic, and political variations.

Adapting the Coping With Caregiving (CWC) program developed by Gallagher-Thompson et al ${ }^{14-17}$ a pilot study was carried out by $\mathrm{Au}$ et $\mathrm{al}^{18}$ to test if the program worked with Hong Kong Chinese caregivers. The psychoeducation program was found to be effective with Caucasian/White as well as Hispanic/Latino and Chinese American dementia family caregivers in the US. The caregivers participated in 13 weekly training sessions, which taught specific cognitivebehavioral strategies to handle caregiving stress. These included the following:

1. Try to understand the cognitive and behavioral changes of the patient

2. Identify stress in the care of the patient

3. Learn basic relaxation techniques

4. Identify the triggers and consequences of problem behavior to minimize the occurrences

5. Identify and change unhelpful thinking

6. Deploy assertive communication skills for effective task performance

7. Plan and engage in pleasant events

8. Plan for the future.

The pilot study of CWC with Hong Kong Chinese caregivers ${ }^{18}$ revealed various issues for further development of the revised program: 1) the importance of enhancing competence in skills development, 2) enhancing accessibility and sustainability of the intervention, and 3) training of paraprofessionals. These issues are briefly discussed below as background to the present study.

Thirty-seven caregivers were recruited for the pilot randomized controlled trial. Participants were randomized into CWC and a wait-list control group. The wait-list control group did have access to the usual care, which consisted mainly of social work services based in the hospital setting or the social service agency. As compared to the wait-list control group, caregivers who completed the CWC program demonstrated a significant increase in self-efficacy in controlling upsetting thoughts and handling disruptive behavior. Moreover, there was an increase in both problem-focused and emotion-focused strategies. There was also an attrition rate of $27 \%$. Seven of those who withdrew said they found it difficult to take so much time away from home to attend the full program. Three of those who discontinued commented that they felt the program required too much time from them in terms of completing all the exercises. A subsequent focus group was conducted 3 months after the completion of the CWC study with the participating caregivers to review the process of intervention. All caregivers remarked the $\mathrm{CWC}$ was very helpful in enhancing their understanding of the problem behaviors of the care recipient and how these behaviors could cause stress themselves. The caregivers expressed, in particular, that the training in pleasant event scheduling and communication skills were the most helpful in terms of coping strategies, as these components help them to acquire skills to change daily lifes and relationships. For instance, one of the participants remarked,

Coping is not to blame ourselves for not doing enough.

We should have time to engage in pleasant activities and ask other people to share the caregiver responsibility. We should have a schedule for both work and fun.

Furthermore, all participating caregivers remarked that they would benefit from regular and longer term support.

On the other hand, however, all the participants felt it was not always straightforward to separate and analyze the antecedents and consequences of problem behavior and to identify unhelpful thoughts. The review of the CWC face-to-face group program had underscored various issues for revision. First, the focus on enhancing the use of coping skills via behavioral activation (BA) may be more acceptable and helpful than changing dysfunctional thoughts, at least in the earlier stages of intervention. 
Second, the revised program would need to consider ways of overcoming barriers to treatment access for those who had difficulties in attending face-to-face meetings. Third, paraprofessionals could be trained to deliver some treatment modules to increase the potential for sustainability of the program.

\section{Rationale for developing the present study on telephone-administered BA interventions}

Daily pleasant experiences can bring balance between selfcare and caring for others and reinforce the positive aspects of caregiving. Uplifts may change the caregiver's experience by reducing some of the negative impact. ${ }^{19-21}$ However, it is not always easy to incorporate pleasant activities in the daily lives of caregivers due to the long-standing stress. Stressed individuals may lack the skills to engage in positive interactions. Moreover, the potency of former positive reinforcers (ie, things that used to bring pleasure) may have diminished. Lewinsohn's seminal description in 1974 of the behavioral theory of depression underscored depression as a function of both low rates of both response-contingent positive reinforcement and inadequate social skills. ${ }^{19}$ These factors can lead to further withdrawal and avoidance behaviors, creating a downward spiral towards further disruption of healthy lifestyle, biological rhythms, and engagement with the social environment, leading to further exacerbation of the depressive symptoms. With the aim to increase positive response-contingent environmental reinforcement, Lewinsohn's BA model focuses on increasing the frequency and duration of engagement in pleasurable activities in order to improve positive affect and reduce negative effect. ${ }^{19}$ There is considerable empirical support for BA. Findings of several studies involving component analysis suggest that BA as a stand-alone intervention may be just as effective as cognitive therapy to treat depression, and also when it is combined with other cognitive-behavioral components. ${ }^{20}$ Various BA procedures have been used in pleasant event scheduling interventions to increase the engagement of pleasurable activities with caregivers. ${ }^{21,22}$ In an earlier pilot study, our team had modified the pleasant event scheduling module of the CWC manual into a 4-week telephone-administered intervention program and has found the program helpful in reducing depressive symptoms of dementia caregivers through a randomized controlled trial. ${ }^{23}$ Furthermore, Ekers et $\mathrm{al}^{24}$ have demonstrated that it was possible to train paraprofessionals to administer BA effectively with 5 days of training and 1 hour of clinical supervision.
At the same time, caregivers can become increasingly secluded for prolonged periods to tackle caregiving demands that escalate with the progression of the disease. ${ }^{25-27}$ This can make it difficult for them to leave their homes to seek support and services through traditional face-to-face interventions. In order to overcome the barriers of obtaining support for caregivers, health and social care professionals have introduced telephone-based support programs targeted to increase accessibility for dementia caregivers. Telephonebased interventions for dementia caregivers, in fact, have been demonstrated to enhance physical and mental health of the caregivers. ${ }^{28-31}$

The present study extended the earlier trial of the previous 4-week telephone-administered pleasant event scheduling ${ }^{23}$ by including a component on communicating with other family members (apart from the care recipients) to seek their support in caregiving. This study began with psychoeducation before moving onto BA. The present study tested whether telephone-delivered psychoeducation combined with an enhanced BA module had a better effect on the wellbeing of dementia caregivers than psychoeducation alone. The study compared two telephone interventions using a 4-month longitudinal randomized controlled trial. For the first 4 weeks, all participants received the same psychoeducation program via telephone. Then, for the following 4 months, eight biweekly telephone follow-up calls were carried out. To test the effectiveness of a condition of lower intensity than weekly calls, this study employed biweekly telephone calls.

For these eight follow-up calls, participants were randomized into either one of the two following conditions. For the psychoeducation with BA (PsyED-BA) group, participants received eight biweekly sessions of BA practice, focused on both 1) pleasant event scheduling and 2) improving communications. For the psychoeducation only (PsyED only) group, there were eight biweekly sessions of general discussion of psychoeducation and related information. Senior citizens were trained and engaged as paraprofessionals to deliver the BA module to increase the potential for sustainability of the program. Details of the procedures are outlined below.

\section{Methods \\ Participants}

Participants were recruited from the United Christian Hospital and also the Prince of Wales Hospital. The inclusion criteria were as follows: they were caregivers aged 25 or above and must have been caring for a care 
recipient diagnosed with Alzheimer's disease for at least 3 months. They should be the primary caregiver and a spouse, kin (eg, daughter/son or daughter/son-in-law), or sibling of the care recipient. To ensure intervention fidelity, participants who showed signs of severe intellectual deficits, demonstrated suicidal ideation, exhibited evidence of psychotic disorders, or could not read or speak fluent Chinese/Cantonese were excluded. A total of 62 participants were recruited. After taking baseline measures, 31 participants were randomly assigned to the PsyED-BA group and 31 to PsyED only group. Fiftynine participants completed the whole study (Figure 1). Two participants consecutively dropped out from the PsyED-BA group at week 4 and week 12. One of them was admitted to the hospital and the other moved to live in Mainland China. One participant in the PsyED only group dropped out at week 4 as the care recipient was admitted to a residential setting. No significant differences in demographic variables were identified between the two groups. All the care recipients had moderate dementia as indicated by the Clinical Dementia Rating Scale. Details can found in Tables 1 and 2 .

\section{Procedures}

Caregivers were approached at the clinics while accompanying the care recipients for their outpatient appointments. The rationale of the study's intervention was introduced both by a printed pamphlet and also verbally. After obtaining written consent, the caregivers were asked to fill in questionnaires regarding their demographic information, and the outcome measures as time 1 (T1) assessment. All of the participating caregivers were given a printed copy of the psychoeducation program together with an information packet with fact sheets concerning local organizations, community resources, and social and mental issues related to dementia. All participants were contacted by telephone within the same week of the baseline assessment in order to start the psychoeducation program. There were a total of four weekly intervention telephone calls, each lasting about 30 minutes. Upon completion of the fourth session in week 4 , a second assessment of the outcome measures, time 2 (T2), was obtained. T2 assessments were carried out by telephone by research staff not involved in executing the psychoeducation program or the subsequent interventions. For the continued intervention over the next 4 months, participants were then randomly assigned

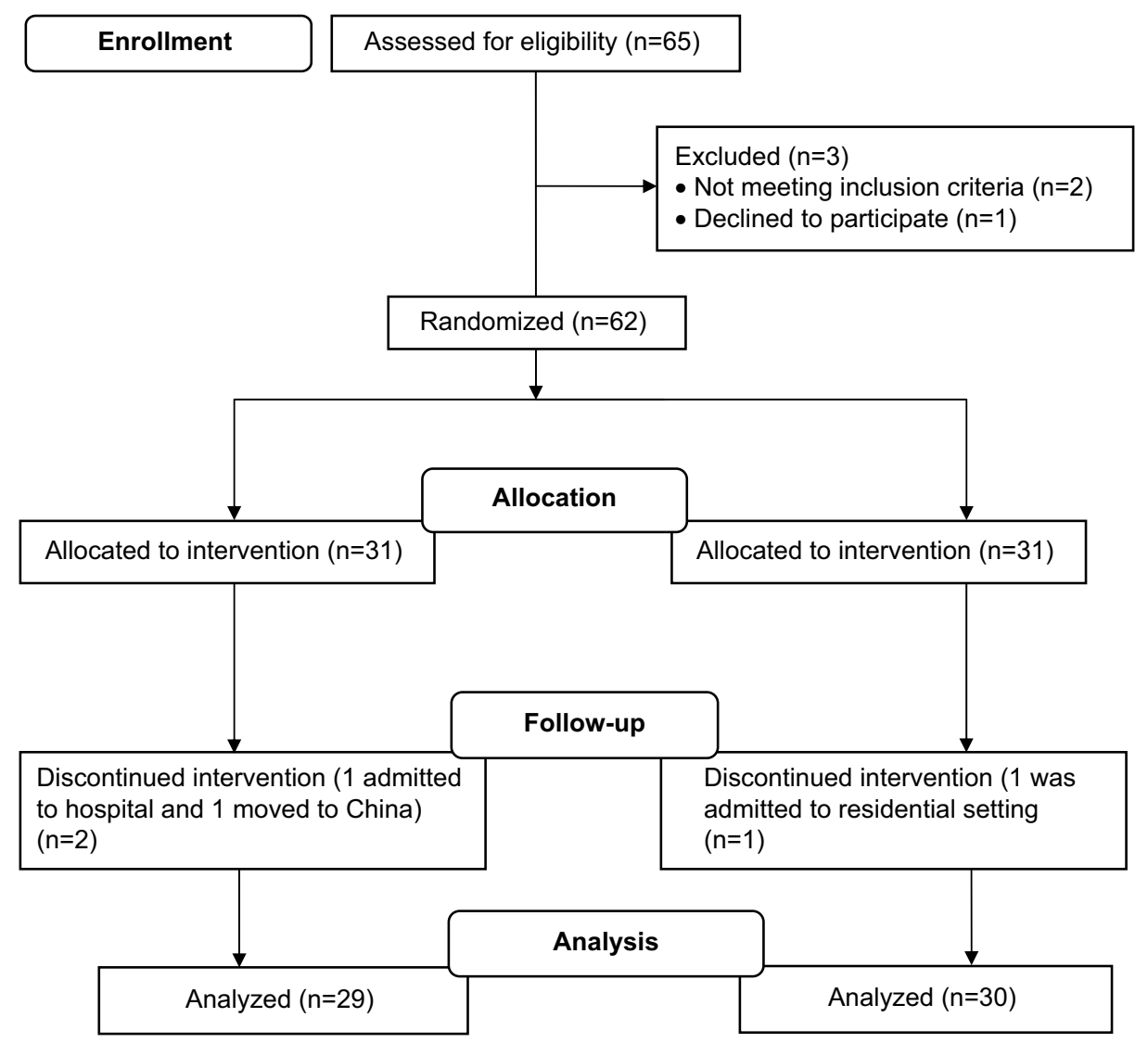

Figure I Flow chart of the study. 
Table I Demographic variables of care recipients $(n=59)$

\begin{tabular}{llllll}
\hline & $\mathbf{n}(\%) / M(S D)$ & $t$ & $\left(\chi^{2}\right)$ & $P$-value \\
\cline { 2 - 4 } & $\begin{array}{l}\text { PsyED-BA } \\
(\mathbf{n}=\mathbf{2 9})\end{array}$ & $\begin{array}{l}\text { PsyED only } \\
(\mathbf{n}=\mathbf{3 0})\end{array}$ & & \\
\hline Age (years) & $81.59(6.35)$ & $79.67(7.48)$ & 1.06 & 0.29 \\
Male/female & $11(37.9 \%) / 18(62.1 \%)$ & $10(33.3 \%) / 20(66.7 \%)$ & & 0.14 \\
MMSE score & $14.61(5.8 I)$ & $16.47(6.17)$ & -1.18 & 0.71 \\
\hline
\end{tabular}

Abbreviations: BA, behavioral activation; M, mean; MMSE, mini-mental state examination; PsyED, psychoeducation; SD, standard deviation.

to one of the two conditions: 1) BA or 2) general discussion about the psychoeducation program and information packet. Time 3 (T3) assessments were carried out within a week after the last call of this 4-month period. Research staff carrying out the assessments and interventions had no knowledge of the allocation. All assessments at the three time points were carried out by research staff not involved in executing the psychoeducation program or the subsequent interventions.

Five paraprofessionals were recruited from the Institute of Active Aging (IAA) at the Hong Kong Polytechnic University and were trained to administer BA procedures. They were aged between 55 and 60 years old and had previously completed postsecondary school training in areas related to human services (eg, nursing or management). They had subsequently completed a 42-hour course on Introduction to Psychology at IAA and had then received 20 hours of group training led by a social worker and a clinical psychologist (the principal investigator of the study) on BA in the context of the CWC program. Each paraprofessional worker was tested on a mock case before delivering the program to actual caregivers. Ongoing weekly supervision was provided by the clinical psychologist and social worker.

\section{Interventions}

All participants took part in the psychoeducation program adapted from the Chinese version of the CWC manual. It was delivered over the telephone individually by a trained social worker. In the first week, all participants were taught about the symptoms and associated behavioral changes of dementia and the possible effects on the caregivers. Participants were also invited to share their caregiving experiences. In the second week, participants learned about the physical, social, and psychological consequences of stress and being aware of their own stress. They were invited to talk about their own stress. In the third week, they learned about the principles of identifying and scheduling pleasant events for themselves. In the fourth week, participants learned about communication: communicating their own needs to seek support from their family members. They also learned about the characteristics of various different types of communications: passive, aggressive, and assertive.

Eight biweekly telephone sessions over the following 4 months were arranged for all participants. Each session consisted of a telephone call lasting 15 to 20 minutes. These were carried out by the paraprofessionals trained for this

Table 2 Demographic variables of caregivers $(n=59)$

\begin{tabular}{|c|c|c|c|c|c|}
\hline & \multicolumn{2}{|l|}{ n (\%)/M (SD) } & \multirow[t]{2}{*}{$t$} & \multirow[t]{2}{*}{$\left(\chi^{2}\right)$} & \multirow[t]{2}{*}{$P$-value } \\
\hline & $\begin{array}{l}\text { PsyED-BA } \\
(n=29)\end{array}$ & $\begin{array}{l}\text { PsyED only } \\
(n=30)\end{array}$ & & & \\
\hline Age (years) & $55.21(8.75)$ & $57.93(12.46)$ & 0.73 & & 0.34 \\
\hline Male/female & $2(6.9 \%) / 27(93.1 \%)$ & $8(26.7 \%) / 22(73.3 \%)$ & & 4.01 & 0.05 \\
\hline Relationship with the patients & & & & 5.35 & 0.15 \\
\hline Spouses & $6(20.7 \%)$ & $12(40 \%)$ & & & \\
\hline Children & $18(62.1 \%)$ & 17 (56.7\%) & & & \\
\hline Relatives & $3(10.3 \%)$ & & & & \\
\hline Daughter/son in laws & $2(6.9 \%)$ & I (3.3\%) & & & \\
\hline $\begin{array}{l}\text { Living distance between caregiver } \\
\text { and care recipient }\end{array}$ & & & & 6.61 & 0.16 \\
\hline Live together & $18(62.1 \%)$ & $25(83.3 \%)$ & & & \\
\hline Care-giving experience (years) & $3.17(2.33)$ & $3.10(1.71)$ & 0.15 & & 0.86 \\
\hline Frequency of care-giving (hours/day) & $8.82(8.09)$ & $10.65(9.23)$ & 0.76 & & 0.45 \\
\hline
\end{tabular}

Abbreviations: BA, behavioral activation; M, mean; PsyED, psychoeducation; SD, standard deviation. 
specific purpose. For the PsyED-BA group, the first four sessions focused on pleasant event scheduling and the other four sessions on effective communication. The tasks involved in each of the four sessions were as follows:

1. Activity monitoring: how is the participant spending time/ communicating now?

2. Activity scheduling: schedule pleasant event/effective communications

3. Reinforcing or modifying the pleasant event and communication based on feedback or self-evaluation

4. Activity rescheduling/revision based on changes after modification.

For the PsyED only group, participants were asked to go through the materials of the psychoeducation program and the information package. For each of the telephone session, the participants were asked to select their own topics for general discussion. If the caregiver selected pleasant event scheduling or communications, general discussion would be carried out without any BA procedures. Finally, for both the PsyED-BA and PsyED only group, social work services were available upon requests in outpatient departments in which the care recipients received their regular follow-up.

\section{Measures}

There were two outcome measures (Table 3 ). The primary outcome was depressive symptoms, and the secondary outcome was satisfaction with relationship with the care recipient. They were chosen because the pleasant event scheduling was intended to improve mood, and the communication skills were intended to improve quality of relationship. Depressive symptoms were assessed by the 20-item Center for Epidemiologic Studies Depressive Scale (CES-D). ${ }^{32}$ Each response was rated from 0 to 3 on a scale of the frequency of occurrence of symptom. To emphasize current state, the instructions read "How often did you in the past week...?" Higher scores would suggest a higher level of depressive

Table 3 Outcome measures

\begin{tabular}{llll}
\hline Outcome measures & $\mathbf{M}(\mathbf{S D})$ & & \\
\cline { 2 - 4 } & Time I & Time 2 & Time 3 \\
\hline RAS & & & \\
$\quad$ PsyED-BA & $28.31(4.74)$ & $28.14(5.60)$ & $30.00(4.55)$ \\
$\quad \begin{array}{l}\text { PsyED only } \\
\text { CES-D }\end{array}$ & $26.10(5.14)$ & $26.63(4.48)$ & $26.57(5.54)$ \\
$\quad$ PsyED-BA & $13.90(7.62)$ & $11.07(8.26)$ & $10.21(9.27)$ \\
PsyED only & $14.37(8.06)$ & $12.80(9.24)$ & $15.60(9.85)$ \\
\hline
\end{tabular}

Abbreviations: BA, behavioral activation; CES-D, Center for Epidemiologic Studies Depressive Scale; M, mean; PsyED, psychoeducation; RAS, Relationship Assessment Scale; SD, standard deviation. symptoms. The seven-item Relationship Assessment Scale $(\mathrm{RAS})^{33}$ was used to measure relationship satisfaction. Rated on a five-point scale, higher scores would suggest a higher level of satisfaction. For this sample, the Cronbach's Alpha of CES-D was 0.90 and RAS, 0.85 .

\section{Data analysis}

Repeated measures analysis of variance (between-subject, groups: PsyED-BA versus PsyED only; within-subject, time: $\mathrm{T} 1$ versus $\mathrm{T} 2$ versus T3) was used to analyze the group effect and the time effect on CES-D and RAS. The $t$-test was used to identify differences between groups at each time point.

\section{Results}

For the CES-D, the interaction between time and group was significant $\left(F[2,56]=2.32, P<0.05, \eta^{2}=0.15\right)$. For the RAS, the interaction between time and group was also significant $\left(F[2,56]=2.61, P<0.05, \eta^{2}=0.16\right)$. Examining group difference at each time point for CES-D, there is no between-group difference at $\mathrm{T} 1(t[57]=0.23, P=0.82)$ or at $\mathrm{T} 2$ $(t[57]=0.76, P=0.45)$, whereas at T3, the PsyED-BA group reported significantly lower levels of depressive symptoms than the PsyED only group $(t[57]=2.97, P<0.01)$. For RAS, examining group difference at each time point, there is no between-group difference at T1 $(t[57]=1.72, P=0.09)$ or at T2 $(t[57]=1.14, P=0.26)$, whereas at T3, the PsyED-BA group showed significantly higher levels of relationship satisfaction than that in the PsyED only group $(t[57]=2.60, P<0.05)$ (Tables 1 and 2).

Comparing the data across the three time points on CES-D for the PsyED-BA group, the level of depressive symptoms at T1 was higher than that at T2 $(t[28]=2.24, P<0.05)$ (Figure 2; Table 3). T2 and T3 were similar $(t[28]=0.46$, $P=0.65)$. For the PsyED only group, levels of the depressive symptoms were similar at T1 and T2 $(t[29]=0.92, P=0.36)$. The level of symptoms were significantly higher than T3 $(t[29]=3.25, P<0.01)$.

For RAS of the PsyED-BA group, there was no significant difference between T1 and T2 $(t[28]=0.18, P=0.86)$ (Figure 3; Table 3). However, RAS at T3 was significantly higher than that at T2 $(t[28]=2.17, P<0.05)$. For the PsyED only group, there was no difference between $\mathrm{T} 1$ and $\mathrm{T} 2(t[29]=0.83$, $P=0.41)$ or between T2 and T3 $(t[29]=0.10, P=0.92)$.

\section{Discussion}

As compared to the PsyED only group, the PsyED-BA group improved with significantly lowered levels of depressive symptoms on CES-D. Results suggest that there were no 


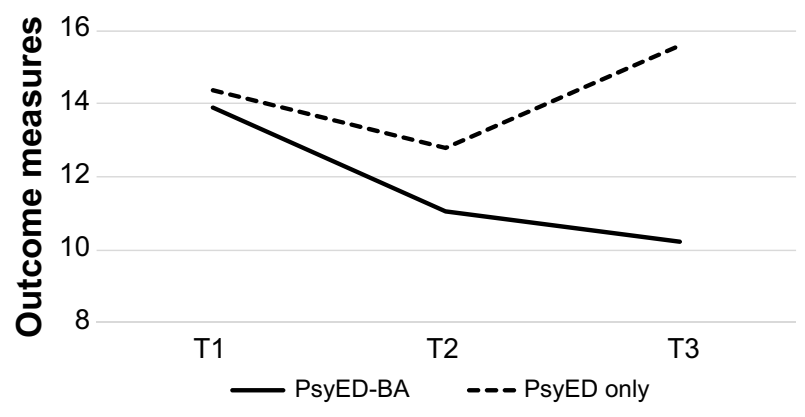

Figure 2 CES-D scores for the PsyED-BA and PsyED only groups on TI, T2, and T3. Abbreviations: BA, behavioral activation; CES-D, Center for Epidemiologic Studies Depressive Scale; PsyED, psychoeducation; T, time.

initial differences between and within groups at T1 and T2 for both groups. However, for the PsyED-BA group, with continued BA intervention between $\mathrm{T} 2$ and $\mathrm{T} 3$, depressive symptoms decreased significantly. On the other hand, for the PsyED only group, with general discussion on PsyED only from T2 to T3, no decrease in depressive symptoms was observed. A similar pattern was observed with the appraisal on caregiver-patient relationship on the RAS; an improvement in relationships from $\mathrm{T} 2$ to $\mathrm{T} 3$ with $\mathrm{BA}$, but this effect was not found in the PsyED only. Comparing the data across the three time points, some significant decrease in CES-D was already achieved from T1 to T2 for the PsyED-BA group, the BA component from $\mathrm{T} 2$ to $\mathrm{T} 3$ consolidated those gains, whereas for the group without the BA component, the level of depressive symptoms increased again to the T1 level. For RAS, no significant differences were achieved for both groups from $\mathrm{T} 1$ to $\mathrm{T} 2$. The BA component, however, helped to achieve gains from $\mathrm{T} 2$ to $\mathrm{T} 3$, where this effect was not found in the PsyED only group. The results suggest that the BA component helped to consolidate the gains made in CES-D and enhanced the gains made in RAS.

Chinese caregivers have also been found to express concern about bothering their family members. ${ }^{25}$ For the present study, in addition to using BA to increase the

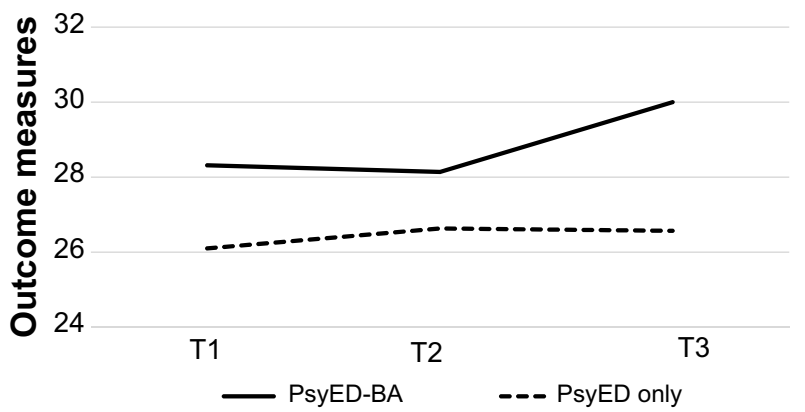

Figure 3 RAS scores for the PsyED-BA and PsyED only groups on TI, T2, and T3. Abbreviations: BA, behavioral activation; PsyED, psychoeducation; RAS, Relationship Assessment Scale; T, time. engagement of pleasurable activities with caregivers, procedures have also been adapted for improving communications based on the CWC manual. Preliminary results suggest that these procedures were helpful in improving mood and relationships. Findings support that training in communications with other family members to seek support can be helpful in enhancing the relationship with the care recipient, possibly due to the increased resourcefulness experienced by the caregiver. BA procedures can be easily tailored to the individual needs of participating caregivers. Starting with self-monitoring exercises, participants can identify their preferred activities and communication patterns as well as reflect on obstacles/barriers to carrying out these activities, and identify means to solve these problems. Subsequent follow-up can help to fine-tune the skills to engage in helpful behaviors. Furthermore, there is increasing evidence that the effects of the emotional stress of caregivers can have a significant effect on the depressive symptomatology of the care recipient. ${ }^{34}$ Thus, further work is needed to address this important area of study, where caregivers and care receivers function as dyads, as the stress associated with caregiving affects each member.

In terms of sustainability and accessibility, Kanter et $\mathrm{a}^{20}$ have highlighted the potential strengths of BA in terms of cost-effectiveness, flexibility, as well as ease of trainability. Ekers et $\mathrm{al}^{24}$ have demonstrated that effective BA, as stepthree high-intensity intervention, can be delivered by general mental health staff after training. Moreover, Soucy Chartier and Provencher ${ }^{35}$ have reviewed several studies supporting the efficacy of BA as a low intensity guided self-help for mild to moderate depression. The results of the study give further support for the potential of BA to be fruitfully engaged as a low-intensity intervention for caregivers of persons living with dementia. In the attempt to enhance sustainability and accessibility, there has been a growing trend to merge evidence-based interventions with the resources offered by community-based paraprofessionals, who can provide practical and emotional support. In particular, productive engagement of older adults has gained attention in recent years. A larger proportion of this age cohort is also likely to remain healthier as they age. More of them will thus be capable of continuing in civic engagement and employment for longer and be able to continue contributing to society. ${ }^{36-38}$ The present study has offered some initial evidence that senior citizens can be successfully engaged as paraprofessional. Results also suggest that BA procedures could be effectively administered with low attrition though telephone contact with the help of senior citizens trained and engaged as paraprofessionals. 
As Chinese caregivers often have concerns about seeking help from outsiders and professionals, the actual implementation of the present program would require a more user-friendly and less stigmatizing venue and medium for promotion and recruitment. Apart from clinical settings, community settings can also be considered. In Hong Kong, for instance, elderly center services, being part of the community support services, render assistance for elders and their caregivers at district and neighborhood levels, facilitating them to receive a multifarious range of services at centers in the vicinity of their homes.

In terms of limitations, the sample size of this study was rather small. Moreover, the psychoeducation component from T1 to T2 of this study only constitutes a period of 4 weeks, with weekly telephone administration lasting for about 30 minutes. The low intensity may not be enough to precipitate therapeutic change. However, for this study, this short-term psychoeducation provided a backdrop for examining the effects of BA. The preliminary evidence from this study does suggest that it is crucial to put into practice what is learned in principle in order to effect a meaningful change in mood and relationships. Future studies should also consider adjusting the length of the psychoeducation component to test variations in effectiveness with a larger sample of participants. Moreover, the procedures might also be considered for caregivers of persons living with other types of chronic illnesses, apart from dementia, as scheduling and communication can be common issues shared by caregivers of persons living with long-term difficulties. Future studies need to consider how treatment gains can be maintained over a longer period through follow-up. Furthermore, the possibilities of extending the present gains to other types of caregiver-patient interactions can be explored. Despite these shortcomings, in the attempt to reach caregivers where they are, findings of this study may contribute to the present literature by offering some framework on how to develop accessible, sustainable, and less costly interventions.

\section{Acknowledgments}

We acknowledge the support of funding by the Food and Health Bureau of the Hong Kong SAR Government (HKPolyU-2011-K-JB3). Part of the study has been presented in Invited Symposium at the 16th Asia Pacific Regional Conference of Alzheimer's Disease International 2013: Capacity Building in Dementia Care held on December 11-13, 2013 in Hong Kong.
We thank Professors Larry Thompson and Dolores Gallagher-Thompson for sharing and allowing us to flexibly adapt the CWC protocol and Ms Sandra Cheng for adapting the materials for telephone administration. We are grateful for the assistance of the senior helpers recruited from the IAA at the Hong Kong Polytechnic University for administrating the follow-up sessions. We also thank all the caregivers and the staff of United Christian Hospital and the Prince of Wales Hospital. The generosity and helpfulness of all those who participated has made this challenging task meaningful and rewarding. We are also grateful to Professor Cheng Sheung Tak from the Hong Kong Institute of Education for his insightful comments on an earlier draft of the paper. Finally, we thank Mrs Teresa Tsien for her inspiring leadership at the Institute of Active Aging of the Hong Kong Polytechnic University.

\section{Disclosure}

The authors report no conflicts of interest in this work.

\section{References}

1. Alzheimer's Disease International. ADI Policy Brief ADI policy brief for the G8 Dementia Summit; 2013.

2. Gallagher-Thompson D, Tzuang YM, Au A, et al. International perspectives on nonpharmacological best practices for dementia family caregivers: a review. Clin Gerontol. 2012;35(4):316-355.

3. Huang CY, Sousa VD, Perng SJ, et al. Stressors, social support, depressive symptoms and general health status of Taiwanese caregivers of persons with stroke or Alzheimer's disease. J Clin Nurs. 2009; 18(4):502-511

4. Ho SC, Chan A, Woo J, Chong P, Sham A. Impact of caregiving on health and quality of life: a comparative population-based study of caregivers for elderly persons and noncaregivers. $J$ Gerontol A Biol Sci Med Sci. 2009;64(8):873-879.

5. Marziali E, McCleary L, Streiner DL. Evaluation of an assessment battery for estimating dementia caregiver needs for health and social care services. Am J Alzheimers Dis Other Demen. 2010;25(5): 446-454.

6. Taylor DH, Kuchibhatla M, Østbye T. Trajectories of caregiving time provided by wives to their husbands with dementia. Alzheimer Dis Assoc Disord. 2008;22(2):131-136.

7. Carbonneau H, Caron C, Desrosiers J. Development of a conceptual framework of positive aspects of caregiving in dementia. Dementia. 2010;9(3):327-353.

8. Pinquart M, Sörensen S. Associations of stressors and uplifts of caregiving with caregiver burden and depressive mood: a meta-analysis. J Gerontol B Psychol Sci Soc Sci. 2003;58(2):P112-P128.

9. Pinquart M, Sörensen S. Associations of caregiver stressors and uplifts with subjective well-being and depressive mood: a meta-analytic comparison. Aging Ment Health. 2004;8(5):438-449.

10. Elliott AF, Burgio LD, Decoster J. Enhancing caregiver health: findings from the resources for enhancing Alzheimer's caregiver health II intervention. $J$ Am Geriatr Soc. 2010;58(1):30-37.

11. Gitlin LN, Belle SH, Burgio LD; REACH Investigators. Effect of multicomponent interventions on caregiver burden and depression: the REACH multisite initiative at 6-month follow-up. Psychol Aging. 2003; 18(3):361-374. 
12. Schulz R, Burgio L, Burns R, et al. Resources for Enhancing Alzheimer's Caregiver Health (REACH): overview, site-specific outcomes, and future directions. Gerontologist. 2003;43(4):514-520.

13. Gitlin LN, Winter L, Burke J, Chernett N, Dennis MP, Hauck WW. Tailored activities to manage neuropsychiatric behaviors in persons in dementia and reduce caregiver burden: a randomized pilot study. Am J Geriatr Psychiatry. 2008;16(3):229-239.

14. Gallagher-Thompson D, Gray HL, Tang PC, et al. Impact of in-home behavioral management versus telephone support to reduce depressive symptoms and perceived stress in Chinese caregivers: results of a pilot study. Am J Geriatr Psychiatry. 2007;15(5):425-434.

15. Gallagher-Thompson D, Solano N, McGee JS, et al. Coping with Caregiving: Reducing Stress and Improving Quality of Life. Stanford University, School of Medicine and VA Palo Alto Care System; 2002.

16. Gallagher-Thompson D, Wang PC, Liu W, et al. Effectiveness of a psychoeducational skill training DVD program to reduce stress in Chinese American dementia caregivers: results of a preliminary study. Aging Ment Health. 2010;14(3):263-273.

17. Gallagher-Thompson D, Tzuang Y, Au A, et al. Families dealing with dementia: insights from China, Hong Kong, and Taiwan. In: Eggleston K, Tuljapurkar S, editors. Aging Asia: Economic and Social Implications of Rapid Demographic Change in China, Japan, and South Korea. Stanford, CA: Stanford University Press/Shorenstein Asia-Pacific Research Center. 2010:157-176.

18. Au A, Li S, Lee K, et al. The Coping with Caregiving Group Program for Chinese caregivers of patients with Alzheimer's disease in Hong Kong. Patient Educ Couns. 2010;78(2):256-260.

19. Lewinsohn PA. A behavioral approach to depression. In Friedman R, Katz M, editors. The Psychology of Depression: Contemporary Theory and Research. New York: Wiley. 1974:157-178.

20. Kanter JW, Puspitasari AJ, Santos MM, Nagy GA. Behavioural activation: history, evidence and promise. Br J Psychiatry. 2012;200(5): 361-363.

21. Losada A, Márquez-González M, Romero-Moreno R. Mechanisms of action of a psychological intervention for dementia caregivers: effects of behavioral activation and modification of dysfunctional thoughts. Int J Geriatr Psychiatry. 2011;26(11):1119-1127.

22. Moore RC, Chattillion EA, Ceglowski J, et al. A randomized clinical trial of Behavioral Activation (BA) therapy for improving psychological and physical health in dementia caregivers: results of the Pleasant Events Program (PEP). Behav Res Ther. 2013;51(10):623-632.

23. Au A, Wong MK, Leung LM, Leung P, Wong A. Telephone-assisted pleasant-event scheduling to enhance well-being of caregivers of people with dementia: a randomised controlled trial. Hong Kong Med J. 2014;20(3 Suppl 3):30-33.
24. Ekers D, Richards D, McMillan D, Bland JM, Gilbody S. Behavioural activation delivered by the non-specialist: phase II randomised controlled trial. Br J Psychiatry. 2011;198(1):66-72.

25. Au A, Shardlow SM, Teng Y, Tsien T, Chan C. Coping strategies and social support-seeing behavior among Chinese caring for older people with dementia. Ageing Soc. 2013;33(8):1422-1441.

26. Au A, Lau KM, Koo S, Cheung G, Pan PC, Wong MK. The effects of informal social support on depressive symptoms and life satisfaction in dementia caregivers in Hong Kong. Hong Kong J Psychiatry. 2009;19:57-64.

27. Au A, Lai MK, Lau KM, et al. Social support and well-being in dementia family caregivers: the mediating role of self-efficacy. Aging Ment Health. 2009;13(5):761-768.

28. van Mierlo LD, Meiland FJ, Dröes RM. Dementelcoach: effect of telephone coaching on carers of community-dwelling people with dementia. Int Psychogeriatr. 2012;24(2):212-222.

29. Tremont G, Davis JD, Bishop DS, Fortinsky RH. Telephone-delivered psychosocial intervention reduces burden in dementia caregivers. Dementia (London). 2008;7(4):503-520.

30. Winter L, Gitlin LN. Evaluation of a telephone-based support group intervention for female caregivers of community-dwelling individuals with dementia. Am J Alzheimers Dis Other Demen. 2007; 21(6):391-397.

31. Grant JR, Steffen AM, Silberbogen AK, Gallagher-Thompson D. Coach Manual: The Dementia Caregiving Skills Program: Reducing Stress and Enjoying Time with Your Family Member. University of Missouri, St Louis/National Institute of Mental Health; 2001. Available from: http:// www.umsl.edu/ steffena/documents/ExcerptFromCoachManual.pdf

32. Radloff LS. The CES-D scale: a self-report depression scale for research in the general population. Appl Psychol Meas. 1977;1(3):385-401.

33. Dicke A, Hendrick C. The relationship assessment scale. J Soc Pers Relat. 1998;15:137-142.

34. Ejem DB, Drentea P, Clay OJ. The effects of caregiver emotional stress on the depressive symptomatology of the care recipient. Aging Ment Health. 2014;28:1-8.

35. Soucy Chartier I, Provencher MD. Behavioural activation for depression: efficacy, effectiveness and dissemination. J Affect Disord. 2013; 145(3):292-299

36. Stevens AB, Lancer K, Smith ER, Allen L, McGhee R. Engaging communities in evidence-based interventions for dementia caregivers. Fam Community Health. 2009;32(1 Suppl):S83-S92.

37. Stevens-Roseman ES, Leung P. Enhancing attitudes, knowledge and skills of paraprofessional service providers in elder care settings. Gerontol Geriatr Educ. 2004;25(1):73-88.

38. Morrow-Howell N, Hong SI, Tang F. Who benefits from volunteering? Variations in perceived benefits. Gerontologist. 2009;49(1):91-102.
Clinical Interventions in Aging

\section{Publish your work in this journal}

Clinical Interventions in Aging is an international, peer-reviewed journal focusing on evidence-based reports on the value or lack thereof of treatments intended to prevent or delay the onset of maladaptive correlates of aging in human beings. This journal is indexed on PubMed Central, MedLine,

\section{Dovepress}

CAS, Scopus and the Elsevier Bibliographic databases. The manuscript management system is completely online and includes a very quick and fair peer-review system, which is all easy to use. Visit http://www.dovepress. com/testimonials.php to read real quotes from published authors. 